\title{
An economic evaluation of community and residential aged care falls prevention strategies in NSW
}

\author{
Jody Church $^{\mathrm{A}, \mathrm{B}}$, Stephen Goodall ${ }^{\mathrm{A}}$, \\ Richard Norman ${ }^{\mathrm{A}}$ and Marion Haas ${ }^{\mathrm{A}}$ \\ ${ }^{\mathrm{A}}$ Centre for Health Economics Research and Evaluation, \\ University of Technology, Sydney \\ ${ }^{\mathrm{B} C o r r e s p o n d i n g ~ a u t h o r . E m a i l: ~ j o d y . c h u r c h @ c h e r e . u t s . e d u . a u ~}$
}

\begin{abstract}
Aim: To evaluate the cost-effectiveness of strategies designed to prevent falls amongst people aged 65 years and over living in the community and in residential aged-care facilities. Methods: A systematic review and meta-analysis of the literature was conducted. The pooled fall rate ratio was used in a decision analytic model that combined a Markov model and decision tree to estimate the costs and outcomes of potential interventions and/or strategies. The resulting cost per quality-adjusted life year was estimated. Results: The most cost-effective falls prevention strategy in community-dwelling older people was Tai Chi. Expedited cataract surgery and psychotropic medication withdrawal were also found to be cost-effective; however, the effectiveness of these interventions is less certain due to small numbers of trials and participants. The most costeffective falls prevention strategies in residential aged-care facilities were medication review and vitamin D supplementation.
\end{abstract}

A fall is defined as 'an unexpected event in which an individual comes to rest on the ground, floor or lower level'. ${ }^{1}$ Falls are common among older people; up to one in four people aged 65 years and over fall at least once in a year, with many falling more than once. Falls are even more common among residents of aged-care facilities, with up to half of all residents falling at least once in a year. ${ }^{2,3}$

Fall-related injury is a major cause of morbidity and mortality for older people. In New South Wales (NSW) each year, falls lead to approximately 30000 hospitalisations and at least 300 deaths in people aged 65 years and over. ${ }^{4}$ Even non-injurious falls can have negative impacts such as depression and mobility restrictions and reduced activities of daily living leading to reductions in quality of life. ${ }^{5,6}$ Projections indicate that without preventative action, and assuming that individuals continue to fall and be injured at the current rate, the costs to the health care system from injurious falls are likely to escalate, reflecting the expected ageing NSW population. The estimated treatment cost associated with falls in NSW is $\$ 558.5$ million, which includes all medical and associated costs occurring in the 12 months following injury and any residential aged-care facility costs beyond the initial fall. ${ }^{7}$

The majority of the economic literature concerning falls prevention strategies use cost per fall avoided as the outcome measure. Consequently, determining whether these interventions are a cost-effective use of resources is problematic since knowledge of society's willingness to pay for an avoided fall is required. A common way of avoiding this complication is to use a generic outcome measure, such as quality-adjusted life years (QALYs). Using cost per QALY gained enables interventions with disparate outcome measures to be compared to a societal value threshold. Using the cost per QALY approach within a randomised controlled trial design, Hendricks et al found a multidisciplinary intervention to be not cost-effective compared to usual care $;{ }^{8}$ Sach et al found cataract surgery in the first eye was not cost-effective ${ }^{9}$ and Busbee et al (using a analytic model design) found cataract surgery in the second eye to be cost-effective. ${ }^{10}$ To date only one study has compared multiple falls prevention interventions in a single study. Frick et al, comparing seven falls prevention interventions, concluded that psychotropic medication withdrawal and Tai Chi were cost-effective interventions. ${ }^{11} \mathrm{~A}$ review of economic evaluations by Davis et al reported three falls prevention strategies to be cost-saving: a multifactorial program; a home-based exercise program for people older than 80 years; and a home safety program for those who have had a previous fall. ${ }^{12}$

A number of strategies have been used to prevent falls and fall-related injuries. These include: group-based exercise; home-based exercise; Tai Chi; vitamin D supplementation; education; clinical medication review; vision and eye examinations; expedited cataract surgery; cardiac 
pacing; psychotropic medication withdrawal; and various multiple and multifactorial interventions representing combinations of the above. The overall aim of this study was to determine which of these interventions is most costeffective using a decision analytic model.

\section{Methods}

A systematic review and meta-analysis were conducted. The results were then used to produce an economic evaluation comparing the costs and outcomes of the aforementioned falls prevention strategies with evidence of effectiveness.

\section{Systematic review and meta-analysis}

A systematic review of the literature was undertaken in September 2008. The objective was to identify interventions that are effective at reducing falls in older people. The review was undertaken prior to the release of the Cochrane review of falls. ${ }^{13}$ Searches were conducted in a number of electronic databases including: PubMed (Medline and PreMedline); EMBASE; the Cochrane Library; the Centre for Reviews and Dissemination databases; Database of Abstracts of Reviews of Effects; National Health Service Economic Evaluation Database; Health Technology Assessment database; and Web of Science. Data were extracted from the included studies by one researcher and checked by a second researcher using standardised extraction tables developed a priori.

Descriptive statistics relating to the number of falls were extracted and rate ratios calculated from each individual trial. A pooled measure of effectiveness was calculated using a random effects model for each falls prevention intervention identified. The analysis was based on an 'intention to treat' principle. Data were entered into Excel and transformed into the required input for statistical analysis. The pooled statistical analysis was conducted using Review Manager 5, a meta-analysis software program available through the Cochrane Collaboration. Only randomised controlled trials with a population mean age of 65 years and over and with more than 20 participants were included in the study. All inclusion/exclusion criteria and a list of all references used in the meta-analysis has been published elsewhere. ${ }^{14}$

During the development of this study, a meta-analysis conducted by the Cochrane Collaboration was released. ${ }^{13}$ The results from the Cochrane review were used for the community-dwelling analysis. Results of both metaanalyses are presented in Table 1 .

\section{Economic evaluation}

A decision analytic model was developed to assess the cost-effectiveness of falls prevention strategies. The rationale for the model is that falls prevention strategies lead to reductions in the number of individuals who fall, which consequently reduces the number of individuals injured or hospitalised due to a fall. Falls resulting in injury and hospitalisation can lead to a reduction in both length of life and quality of life. Consequently, a reduction in the number of fall-related injuries will result in measureable improvements in terms of QALYs.

QALYs are the most common and convenient outcome measure used in economic evaluation. They combine quality of life and life expectancy into one metric, therefore enabling comparison of multiple strategies across different interventions and settings. Such an approach, termed a cost-utility analysis, was adopted in this project. The usual approach to economic evaluation is first to determine the incremental effectiveness and incremental cost and combine these to calculate the incremental costeffectiveness ratio (ICER) using the following ratio:

$$
\mathrm{ICER}=\frac{\left(\text { Cost }_{\mathrm{New}}\right)-\left(\text { Cost }_{\text {Comparator }}\right)}{\left(\text { Effectiveness }_{\mathrm{New}}\right)-\left(\text { Effectiveness }_{\text {Comparator }}\right)}
$$

Model

The decision analytic model was designed to capture the transition of people between various health states. Five Markov states were assigned as follows:

- low risk (individuals who have never fallen)

- medium risk (individuals who have previously fallen but incurred no injury)

- high risk (previously injured individual who fell)

- residential aged care

- death.

The Markov model is summarised in Figure 1. The Markov model was built using TreeAge Pro Suite 2009 and a decision tree was embedded between each Markov state. Within the decision tree, the probability of transitioning to another state depends on the occurrence of various events, such as presenting at the emergency department and being admitted to hospital. Costs and outcomes were incorporated into the model as a mean value per state per cycle. Expected values for costs and outcomes in the intervention and control are calculated by summing the costs and outcomes accrued by everyone in the model under both intervention and comparator, then dividing by the number of people in the model to produce a mean cost and outcome for each intervention. The starting cohort age in the communitydwelling model was 75 years ( 85 years in the residential aged-care facility model), the cycle length of the model was 1 year and costs and benefits were measured over 10 years. All costs and outcomes were discounted at a rate of $5 \%{ }^{15}$

\section{Model inputs}

The data used in the model were obtained from different sources including published literature on falls prevention, 
Table 1. Meta-analysis of interventions to prevent falls in community dwellings and residential aged-care facilities

\begin{tabular}{|c|c|c|c|}
\hline Intervention & $\begin{array}{l}\text { CHERE community- } \\
\text { dwelling results } \\
\text { (rate ratio, } 95 \% \mathrm{Cl} \text { ) }\end{array}$ & $\begin{array}{l}\text { Cochrane community- } \\
\text { dwelling results } \\
\text { (rate ratio, } 95 \% \mathrm{Cl} \text { ) }\end{array}$ & $\begin{array}{l}\text { CHERE residential } \\
\text { aged-care } \\
\text { facilities results } \\
\text { (rate ratio, } 95 \% \mathrm{Cl} \text { ) }\end{array}$ \\
\hline Group-based exercise & $0.79(0.70,0.86)$ & $0.78(0.71,0.86)$ & $0.79(0.56,1.11)$ \\
\hline Home-based exercise & $0.81(0.58,1.14)$ & $0.66(0.53,0.82)$ & - \\
\hline Tai Chi & $0.66(0.57,0.77)$ & $0.63(0.52,0.78)$ & $0.96(0.79,1.17)$ \\
\hline Vitamin D supplementation & $0.95(0.85,1.07)$ & $0.95(0.80,1.14)$ & $0.86(0.83,0.90)$ \\
\hline Education & $0.85(0.73,1.00)$ & - & - \\
\hline Home hazard assessment and modification & $0.73(0.52,1.03)$ & $0.90(0.79,1.03)$ & - \\
\hline Psychotropic medication withdrawal & $0.40(0.23,0.70)$ & $0.34(0.16,0.73)$ & - \\
\hline Clinical medication review & Not estimable & Not estimable & $0.59(0.49,0.70)$ \\
\hline Expedited cataract surgery & $0.66(0.49,0.90)$ & $0.66(0.45,0.75)$ & - \\
\hline Vision and eye exam & $1.57(1.39,1.76)$ & - & - \\
\hline Cardiac pacing & $0.33(0.28,0.38)$ & $0.42(0.23,0.75)$ & \\
\hline Multiple interventions ${ }^{a}$ & $0.80(0.70,0.90)$ & $0.69(0.50,0.96)^{b}$ & $0.76(0.59,0.97)$ \\
\hline Exercise and home hazard & $0.76(0.65,0.90)$ & - & - \\
\hline Exercise and falls advice & $0.86(0.71,1.03)$ & - & - \\
\hline Exercise and supplementation & $0.57(0.27,1.20)$ & - & - \\
\hline Multifactorial interventions ${ }^{\mathrm{b}}$ & $0.72(0.62,0.83)$ & $0.75(0.65,0.86)$ & $0.76(0.59,0.97)$ \\
\hline Assessment and referral & $0.81(0.72,0.92)$ & $0.84(0.72,0.98)$ & $1.34(1.06,1.69)$ \\
\hline Assessment and active intervention & $0.67(0.52,0.85)$ & $0.70(0.55,0.90)$ & $0.68(0.53,0.87)$ \\
\hline \multicolumn{4}{|c|}{$\begin{array}{l}\text { Only interventions that were statistically significant (bold) were modelled. } \\
\text { CHERE = Centre for Health Economics Research and Evaluation. } \\
\text { a'Stepping on Program' includes exercise program, falls prevention education and occupational therapy home visit. In the Cochrane review, } \\
\text { Swanenburg }{ }^{28} \text { was also found to be significant in favour of falls reduction, but was not modelled due to the low number of participants. } \\
\text { In the economic model the rate ratio was derived from the Cochrane review. } \\
\text { b Include a multifactorial assessment and a referral for further treatment based on individual participant scoring. Those with an active component } \\
\text { (e.g. an exercise program or occupational therapy visit) were modelled separately. }\end{array}$} \\
\hline
\end{tabular}

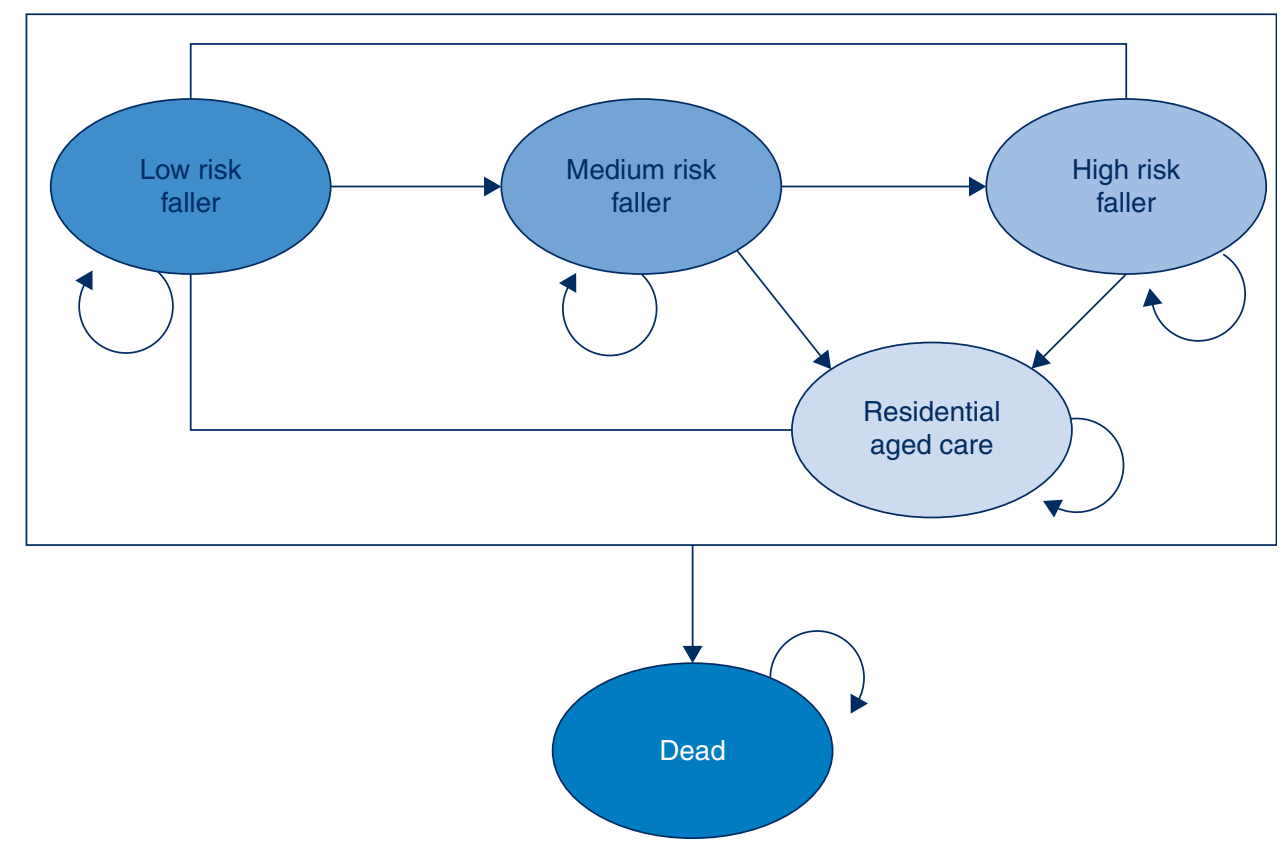

Figure 1. Community-dwelling Markov model showing the pathway between faller risk group, residential aged-care facilities and death. 
expert opinion, the Australian Bureau of Statistics, the Australian Institute of Health and Welfare, and reports released by the NSW Government, specifically a report by Dr Wendy Watson from the Injury Risk Management Research Centre at the University of NSW. ${ }^{7}$ In the absence of suitable data, assumptions were made and tested in the model. Specific details about the derivation of inputs are presented below. Further details have been published elsewhere. $^{14}$

Table 2. Probability of falling adjusted for prior history of falling and age group

\begin{tabular}{|c|c|c|c|}
\hline $\begin{array}{l}\text { Age group } \\
\text { (years) }\end{array}$ & Low risk $^{a}$ & Medium risk ${ }^{b}$ & High risk $^{a}$ \\
\hline \multicolumn{4}{|c|}{ Community-dwelling } \\
\hline 65-69 & 0.18 & 0.25 & 0.39 \\
\hline $70-74$ & 0.18 & 0.25 & 0.39 \\
\hline $75-79$ & 0.23 & 0.35 & 0.50 \\
\hline $80-84$ & 0.26 & 0.40 & 0.57 \\
\hline $85+$ & 0.31 & 0.50 & 0.68 \\
\hline \multicolumn{4}{|c|}{ Residential aged care } \\
\hline $65-69$ & 0.26 & 0.36 & 0.57 \\
\hline $70-74$ & 0.26 & 0.36 & 0.57 \\
\hline $75-79$ & 0.32 & 0.50 & 0.72 \\
\hline $80-84$ & 0.37 & 0.57 & 0.82 \\
\hline $85+$ & 0.44 & 0.71 & 0.97 \\
\hline \multicolumn{4}{|c|}{$\begin{array}{l}\text { aBased on estimate that the absolute risk of being a faller if you fell in } \\
\text { the past year was } 71 \% \text { and } 32 \% \text { if you had not fallen in the past year. } \\
\text { bPersonal communication from Professor Stephen Lord. } \\
\text { Low risk = no previous fall, Medium risk = previously fallen but did } \\
\text { not suffer a serious injury, High risk = previously fallen and suffered a } \\
\text { previous injury that required hospitalisation. }\end{array}$} \\
\hline
\end{tabular}

\section{Transition probabilities}

The initial population distributions for the low, medium and high risk states were derived from Lord et al (1993) and the probability of falling was derived by Lord, broken down by age (Table 2). ${ }^{16}$ The transition probabilities to emergency, other medical, hospital, residential aged-care facilities, respite care or death due to a fall were taken from the report by Watson et al (2009) (Table 3). ${ }^{7}$ The Australian Bureau of Statistics life tables ${ }^{17}$ were used to obtain data on all cause mortality and the probability of entering residential aged-care facilities from 'all causes' was estimated from a study by Wang et al (2001). ${ }^{18}$

\section{Effectiveness}

The effectiveness of each intervention was based on the pooled rate ratio obtained from the Centre for Health Economics Research and Evaluation meta-analysis and the Cochrane review. ${ }^{13}$ Only interventions with a statistically significant reduction in the risk of falling were included in the model. For the community-dwelling population, the results from the Cochrane review were used unless otherwise specified. The results from the metaanalysis conducted during this study were used for the residential aged care population. The estimated rate ratio was used to adjust the probability of falling for each intervention compared to no intervention. In the model, each time an individual falls, the rate ratio was increased by a fall rate multiplier to take into account that individuals may fall multiple times in any given year.

Each intervention is described below. Further details of each intervention have been published elsewhere. ${ }^{14}$

- Group-based exercise - two group classes and one home exercise session per week for 26 weeks.

Table 3. Transition probabilities post fall, adjusted for age

\begin{tabular}{|c|c|c|c|c|c|c|}
\hline $\begin{array}{l}\text { Age group } \\
\text { (years) }\end{array}$ & Emergency & $\begin{array}{l}\text { Other } \\
\text { medical }\end{array}$ & $\begin{array}{l}\text { Admitted to } \\
\text { hospital }\end{array}$ & $\begin{array}{c}\text { Death due to } \\
\text { a fall }\end{array}$ & $\begin{array}{l}\text { Discharge to } \\
\text { residential } \\
\text { aged-care facility }\end{array}$ & $\begin{array}{l}\text { Discharge to } \\
\text { respite care }\end{array}$ \\
\hline \multicolumn{7}{|c|}{ Community-dwelling } \\
\hline $65-69$ & 0.09 & 0.13 & 0.26 & 0.008 & 0.008 & 0.004 \\
\hline $70-74$ & 0.14 & 0.21 & 0.18 & 0.01 & 0.02 & 0.01 \\
\hline $75-79$ & 0.18 & 0.22 & 0.19 & 0.02 & 0.03 & 0.02 \\
\hline $80-84$ & 0.25 & 0.31 & 0.28 & 0.03 & 0.05 & 0.03 \\
\hline $85+$ & 0.35 & 0.31 & 0.30 & 0.06 & 0.09 & 0.04 \\
\hline \multicolumn{7}{|c|}{ Residential aged care } \\
\hline 65-69 & 0.04 & 0.44 & 0.57 & 0.01 & - & - \\
\hline $70-74$ & 0.07 & 0.40 & 0.46 & 0.01 & - & - \\
\hline $75-79$ & 0.10 & 0.39 & 0.46 & 0.02 & - & - \\
\hline $80-84$ & 0.13 & 0.32 & 0.44 & 0.03 & - & - \\
\hline $85+$ & 0.15 & 0.27 & 0.44 & 0.06 & - & - \\
\hline
\end{tabular}

Source: Watson W, Clapperton A, Mitchell R. The cost of fall injuries among older people in NSW, 2006-07. NSW Injury Risk Management Research Centre, University of New South Wales; 2009. 
Table 4. Cost of falls prevention interventions

\begin{tabular}{|c|c|c|}
\hline Intervention & $\begin{array}{c}\text { Costs } \\
\$\end{array}$ & Source \\
\hline \multicolumn{3}{|l|}{ Community-dwelling interventions } \\
\hline Group-based exercise & 563 & Estimated from Sherrington et $\mathrm{al}^{29}$ \\
\hline Home-based exercise & 1091 & Day et $\mathrm{al}^{18}$ \\
\hline Tai Chi & 648 & Estimated from Sherrington et $\mathrm{al}^{29}$ \\
\hline Psychotropic medication withdrawal & 604 & Day et $a^{19}$ \\
\hline Cardiac pacing & 13526 & Day et $\mathrm{al}^{19}$ \\
\hline Expedited cataract surgery & 2050 & DRG hospital data, MBS code 23, MBS code 10900 \\
\hline Multiple intervention & 785 & NSW Health personal correspondence and Clemson et al ${ }^{30}$ \\
\hline Multifactorial - assessment and referral & 855 & Day et $\mathrm{al}^{19}$ \\
\hline Multifactorial - assessment and active & 1244 & Day et $\mathrm{al}^{19}$ \\
\hline \multicolumn{3}{|l|}{ Residential aged care interventions } \\
\hline Vitamin D & 138 & www.pharmacyonline.com.au \\
\hline Clinical medication review & 228 & MBS code 903, Pharmacy Guild \\
\hline Multiple intervention & 775 & $\begin{array}{l}\text { Based on Becker et al, }{ }^{31} \text { Department of Veterans' Affairs, Vitamin D } \\
\text { (as above), Kainos printing, NSW Health Award Wage rates }\end{array}$ \\
\hline Multifactorial - assessment and active & 1244 & Day et $\mathrm{al}^{19}$ \\
\hline
\end{tabular}

- Home-based exercise - five district nurse home visits in the first week, followed by home visits at week 2, 4 and 8 weeks with a booster at 6 months. Costs include nurse and physiotherapist time.

- Tai Chi - 6-month instructed classes twice a week for 12 participants.

- Expedited cataract surgery - patients receive the cataract procedure within 4 weeks versus the usual 12-month waiting period. Costs include a general practitioner (GP) visit, surgery and two specialist visits.

- Cardiac pacing - screening by carotid sinus massage, cardiovascular assessment, insertion of a pacemaker and post-pacemaker visit.

- Psychotropic medication withdrawal - reduction of medication over 14 weeks with six GP visits and nurse time to check register.

- Multiple - based on the Stepping On Program. Two-hour weekly group information sessions on falls prevention run by an occupational therapist for 7 weeks with a follow-up home visit and a 3-month booster.

- Multifactorial (referral only) - falls risk assessment and follow-up by a physician, 1-hour occupational therapy home visit and a 2-hour nurse interview.

- Multifactorial (referral and active) - falls risk assessment (as per above) plus an exercise program once a week, home hazard modification by an occupational therapist, a vision assessment, a medication review (as per above) and counselling.

- Vitamin D supplementation (residential aged-care facility model) - daily dose of Vitamin D (1000 IU) plus $600 \mathrm{mg}$ of calcium.

- Clinical medication review (residential aged-care facility model) - medication management review by a GP and a residential medication management review by an accredited pharmacist.
- Multiple intervention (residential aged-care facility model) - physiotherapist visit, consultation with a residential aged care nurse, falls prevention information, occupational therapist visit, participation in a group-exercise program and hip protectors.

- Multifactorial intervention (residential aged-care facility model) - falls risk assessment and follow-up visit by a physician, hip protectors and hazard modifications by an occupational therapist.

\section{Costs}

The cost of each intervention was estimated from the published literature (if available), personal correspondence with NSW Health and online sources (Table 4). The majority of intervention costs were obtained from Day et al. ${ }^{19}$ All health-care-related costs were obtained using Watson et al. ${ }^{7}$ All health-care-related costs were inflated to 2009 costs using the average health price index for government expenditure on hospitals and nursing homes from the Australian Institute of Health and Welfare report Health expenditure Australia 2008-09. ${ }^{20}$

\section{Utility}

The baseline utility estimates used in the model were based on the UK Population Norms using the EQ-5D. ${ }^{21}$ The EQ-5D is a generic measure of health status where 0 represents death and 1 represents full health. For example, the average utility of a individual aged 75 years is 0.731 . In the model, a utility decrement was incurred once an individual attended an emergency department (-0.014), was admitted to hospital $(-0.144)$ or entered residential aged care $(-0.06)$. Individuals who received a fracture and attended hospital also received a utility decrement in each of the 
Table 5. Community-dwelling: incremental cost per fall avoided, hospitalisation avoided and QALY gained

\begin{tabular}{|c|c|c|c|}
\hline Intervention & $\begin{array}{l}\text { Incremental cost } \\
\text { per fall avoided } \\
(\$ / \text { fall })\end{array}$ & $\begin{array}{l}\text { Incremental cost per } \\
\text { hospitalisation avoided } \\
\text { (\$/hospitalisation) }\end{array}$ & $\begin{array}{c}\text { Incremental cost } \\
\text { per QALY } \\
\text { (\$/QALY) }\end{array}$ \\
\hline \multicolumn{4}{|l|}{ General population } \\
\hline Tai Chi & 239 & 5172 & 44879 \\
\hline Group-based exercise & 4925 & 104318 & 72765 \\
\hline Multiple - Stepping On Program & 5957 & 129231 & 74186 \\
\hline Home-based exercise & 1987 & 41944 & 96205 \\
\hline Multifactorial - active & 9774 & 216250 & 130139 \\
\hline Multifactorial - referral & 2270 & 47586 & 172009 \\
\hline \multicolumn{4}{|l|}{ Specific populations } \\
\hline Expedited cataract surgery & 153 & 3281 & 2211 \\
\hline Psychotropic medication withdrawal & 1123 & 24048 & 16584 \\
\hline Cardiac pacing & 4545 & 68167 & 80257 \\
\hline
\end{tabular}

Table 6. Residential aged care: incremental cost per fall avoided, hospitalisation avoided and QALY gained

\begin{tabular}{lccc}
\hline Intervention & $\begin{array}{c}\text { Incremental cost per } \\
\text { fall avoided } \\
(\$ / \text { fall) }\end{array}$ & $\begin{array}{c}\text { Incremental cost per } \\
\text { hospitalisation avoided } \\
\text { (\$/hosp) }\end{array}$ & $\begin{array}{c}\text { Incremental cost } \\
\text { per QALY } \\
(\$ / Q A L Y)\end{array}$ \\
\hline Medication review & Dominant & Dominant & Dominant \\
Vitamin D & 6 & 80 & 106 \\
Multiple - exercise and home hazard modification & 2658 & 33993 & 45287 \\
Multifactorial - active & 3334 & 42648 & 56752 \\
\hline
\end{tabular}

subsequent years following a fall $(-0.072)$. These utility measures were estimated from a variety of published literature based on the utility loss of a wrist fracture, vertebral fracture, hip fracture, previous fracture and residential aged care. ${ }^{21-25}$ A utility decrement for the fear of falling $(-0.045)$ was also included in the model regardless of any injury or hospitalisation; this estimate was obtained from Iglesias et al. ${ }^{26}$ Loss in utility due to fear of falling was calculated as a weighted proportion from three studies that categorised fear of falling over six categories: none of the time; a little of the time; some of the time; a good bit of the time; most of the time; and all of the time.

\section{Results}

Each of the interventions was analysed over a 10-year period for a cohort of individuals aged 75 years. This is the average age of those older than 65 years in NSW and was used as our base case. The base case for the residential aged-care facility model was individuals aged 85 years. Exercise, Tai Chi, psychotropic medication withdrawal and multiple and multifactorial interventions were all assumed to incur both costs and benefits of the intervention in year one only. Expedited cataract surgery and cardiac pacing were assumed to incur costs in year one only, but the benefits would be experienced for as long as the model was run. Interventions such as vitamin D supplementation and medication review were assumed to be ongoing interventions and both the costs and benefits would be incurred for as long as the model was run.

Outcomes from the model were measured in terms of falls avoided, hospitalisations avoided and QALYs gained. Tables 5 and 6 summarise the cost-effectiveness results which show the additional costs and benefits of providing the intervention. This includes the actual cost of providing the intervention minus the cost of avoided medical treatment due to falls averted. In this respect, the 'do nothing' option against which all interventions are compared is not costless because this option incurs the maximum fallrelated treatment costs.

\section{Sensitivity analysis}

Using group-based exercise as an example (with the comparator defined as no intervention), each possible parameter was tested in a sensitivity analysis. Where 


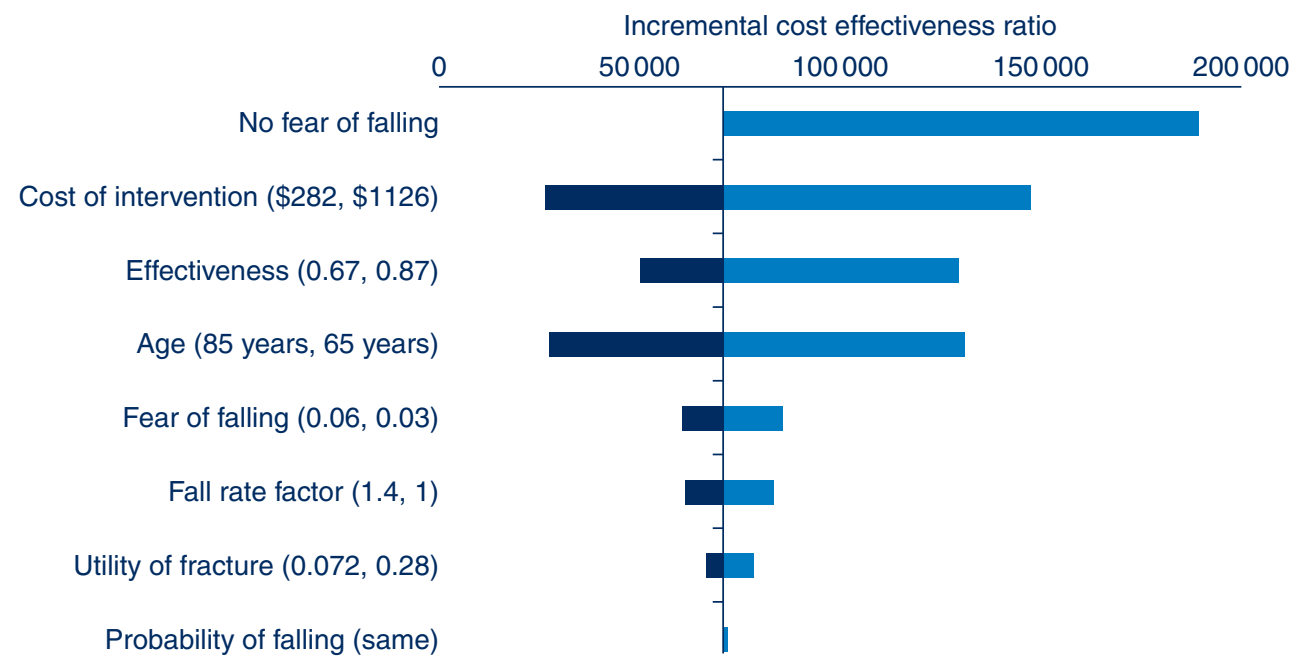

Figure 2. Sensitivity analysis: tornado plot of group-based exercise versus no intervention (base case cost per QALY gained $=\$ 72765$ ).

possible, the confidence interval was used as the range for the analysis; if this was not available, the best estimate of possible ranges was used or the parameter was adjusted up and down by $25 \%$. A tornado plot illustrating the results of the sensitivity analysis is presented in Figure 2. The fear of falling is the main driver of the model. This is expected as each time a fall is avoided the QALY decrement associated with a fall is also avoided. The effectiveness of the intervention and cost of the intervention are also drivers in the model.

\section{Discussion}

Incremental cost per fall avoided or hospitalisation avoided were presented in this analysis. However, using surrogate outcomes such as these makes it difficult to judge whether an intervention represents value for money in terms of the total health care budget. In order to make this decision it is necessary to either value society's willingness to pay to avoid a fall or hospitalisation; or alternatively, a generic outcome measure, such as life years gained or QALYs gained, can be used. The advantage of using the latter approach is that interventions targeting different health conditions (not just falls prevention) can be compared and the most cost-effective interventions can be adopted. The results presented in this analysis compare each intervention to the 'do nothing' option.

Currently there is no cost-effectiveness threshold in Australia. An implicit threshold of between $\$ 50000$ and $\$ 60000$ per QALY gained is often mentioned as being appropriate. ${ }^{27}$ However, this threshold relates to pharmaceutical products and consequently may not be suitable for falls prevention strategies. Yet, if this threshold represents society's willingness to pay for a QALY gained, the following community-dwelling interventions would be considered cost-effective: expedited cataract surgery; psychotropic medication withdrawal; and Tai Chi.
Group-based exercise and a multiple intervention combining exercise, education and occupational therapy would be approaching cost-effectiveness. The following residential aged-care interventions would be considered cost-effective: medication review; and vitamin D supplementation and a multiple intervention.

There are some limitations to this analysis. The point estimates used in the economic model are based on a meta-analysis of falls prevention interventions. Therefore, they do not take account of the heterogeneity of similar interventions. For example, the pooled cost and pooled effectiveness estimates may mask the fact that high cost interventions (e.g. exercise three times a week compared to once a week) are more effective relative to the cheaper equivalent. Also, while using a random effects model may widen the confidence intervals around a particular parameter, it does not explain the heterogeneity that exists between the studies. The pooling of study results may not be the most appropriate means of deriving an estimate of effectiveness. Furthermore, the costs of the interventions are estimated based on mean duration and intensity, hence a higher cost intervention could indicate a more effective intervention.

The evidence for the effectiveness of some interventions is based on limited data from a small number of studies or studies with few participants. Sensitivity analysis has been conducted to test any uncertainty but caution is still warranted in interpreting the results. Another consideration is that some interventions are targeted at specific patient groups; therefore extrapolating the effectiveness results to a general population may yield different results. Finally, most studies quote a $30 \%$ fall rate in the community and $50 \%$ fall rate in residential aged care. However, further research is required to determine the exact fall rate in different age groups and by fall risk profile. 


\section{Conclusion}

The most cost-effective falls prevention strategy in community-dwelling older people was Tai Chi. Expedited cataract surgery and psychotropic medication withdrawal were also found to be cost-effective in specific populations however the effectiveness of these interventions is less certain due to small numbers of trials and participants. The most cost-effective falls prevention strategies in residential aged care were medication review and vitamin $\mathrm{D}$ supplementation.

\section{Acknowledgments}

The authors would like to thank NSW Health for their contribution to the project, as well as Professor Stephen Lord from the Prince of Wales Medical Research Institute and Dr Wendy Watson from the Injury Risk Management Research Centre. We are also indebted to the advisory committee members for their input in this process including: Rebecca Mitchell, Andrew Milat, Joanne Smith, Claire Monger and Lorraine Lovitt.

\section{References}

1. Lamb SE, Jorstad-Stein EC, Hauer K, Becker C. Development of a common outcome data set for fall injury prevention trials: the Prevention of Falls Network Europe consensus. $J$ Am Geriatr Soc 2005; 53(9): 1618-22. doi:10.1111/j.15325415.2005.53455.x

2. NSW Department of Health. Report on Adult Health from the New South Wales Population Health Survey. North Sydney: NSW Department of Health; 2007.

3. Norton R, Butler M. Prevention of Falls and fall-related injuries among institutionalised older people. Wellington: University of Auckland; 1997.

4. NSW Department of Health 2010. Available from: http://www. gsahs.nsw.gov.au/page.asp?t=mr\&p=1497 (Cited 1 June 2010.)

5. Boyd R, Stevens JA. Falls and fear of falling: burden, beliefs and behaviours. Age Ageing 2009; 38(4): 423-8. doi:10.1093/ ageing/afp053

6. Masud T, Morris RO. Epidemiology of falls. Age Ageing 2001; 30(Suppl. 4): 3-7.

7. Watson W, Clapperton A, Mitchell R. The cost of fall injuries among older people in NSW, 2006-07. NSW Injury Risk Management Research Centre, University of New South Wales; 2009.

8. Hendriks MRC, Evers SMAA, Bleijlevens MHC, van Haastreg JCM, Crebolder HFJM, van Eijk JTM. Cost-effectiveness of a multidisciplinary fall prevention program in communitydwelling elderly people: A randomized controlled trial (ISRCTN 64716113). Int J Technol Assess Health Care 2008; 24(2): 193-202. doi:10.1017/S0266462308080276

9. Sach TH, Foss AJ, Gregson RM, Zaman A, Osborn F, Masud T et al. Falls and health status in elderly women following first eye cataract surgery: an economic evaluation conducted alongside a randomised controlled trial. Br J Ophthalmol 2007; 91(12): 1675-9. doi:10.1136/bjo.2007.118687

10. Busbee BG, Brown MM, Brown GC, Sharma S. Cost-utility analysis of cataract surgery in the second eye. Ophthalmology 2003; 110(12): 2310-7. doi:10.1016/S0161-6420(03)00796-6
11. Frick KD, Kung JY, Parrish JM, Narrett MJ. Evaluating the costeffectiveness of fall prevention programs that reduce fall-related hip fractures in older adults. $J$ Am Geriatr Soc 2010; 58(1): 136-41. doi:10.1111/j.1532-5415.2009.02575.x

12. Davis JC, Robertson MC, Ashe MC, Liu-Ambrose T, Khan KM, Marra CA. Does a home based strength and balance programme in people aged $>=80$ years provide the best value for money to prevent falls?: A systematic review of economic analyses of falls prevention interventions. Br J Sports Med 2010; 44(2): 80-9. doi:10.1136/bjsm.2008.060988

13. Gillespie LD, Robertson MC, Gillespie WJ, Lamb SE, Gates S, Cumming RG et al. Interventions for preventing falls in older people living in the community. Cochrane Database Syst Rev 2009; 2: CD007146.

14. Church J, Goodall S, Norman RP, Haas MR. An economic evaluation of community and residential aged care falls prevention strategies in NSW. A report by the Centre for Health Economics Research and Evaluation as part of the Costing for Health Economic Evaluation Program (CHEEP). Sydney: Centre for Health Economics Research and Evaluation; 2010. Available from: http://www.chere.uts.edu.au/pdf/falls.pdf (Cited 5 April 2011.)

15. Department of Health and Ageing. Guidelines for preparing submissions to the pharmaceutical benefits advisory committee (version 4.2). Available from: http://www.health.gov.au/ internet/main/publishing.Nsf/content/pbacguidelines-index (Cited 1 June 2010.)

16. Lord SR, Ward JA, Williams P, Anstey KJ. An epidemiological study of falls in older community-dwelling women: the Randwick falls and fractures study. Aust J Public Health 1993; 17(3): 240-5. doi:10.1111/j.1753-6405.1993.tb00143.x

17. Australian Bureau of Statistics. Life Tables Australia 2005 2007, Catalogue No. 3302055001DO001_20052007. Canberra: Commonwealth of Australia; 2008.

18. Wang JJ, Mitchell P, Smith W, Cumming RG, Leeder SR. Incidence of nursing home placement in a defined community. Med J Aust 2001; 174(6): 271-5.

19. Day L, Hoareau E, Finch C, Harrison J, Segal L, Bolton T et al. Modelling the impact, costs and benefits of falls prevention measures to support policy-makers and program planners. Monash University Accident Research Centre. Available from: http://www.monash.edu.au/muarc/reports/muarc286.pdf (Cited 1 June 2010.)

20. Australian Institute of Health and Welfare. Health expenditure Australia 2008-09. Health and welfare expenditure series no. 42. Cat. no. HWE 51. Canberra: AIHW; 2010.

21. Brazier JE, Green C, Kanis JA. A Systematic Review of Health State Utility Values for Osteoporosis Related Conditions. Osteoporos Int 2002; 13: 768-76. doi:10.1007/ s001980200107

22. Honkanen LA, Mushlin AI, Lachs M, Schackman BR. Can hip protector use cost-effectively prevent fractures in communitydwelling geriatric populations? J Am Geriatr Soc 2006; 54(11): 1658-65. doi:10.1111/j.1532-5415.2006.00939.x

23. Peel N, Steinberg M, Williams G. Home safety assessment in the prevention of falls among older people. Aust N ZJ Public Health 2000; 24(5): 536-9. doi:10.1111/j.1467-842X.2000. tb00506.x

24. Strom O, Borgstrom F, Sen SS, Boonen S, Haentjens P, Johnell $\mathrm{O}$ et al. Cost-effectiveness of alendronate in the 
treatment of postmenopausal women in 9 European countries an economic evaluation based on the fracture intervention trial. Osteoporos Int 2007; 18(8): 1047-61. doi:10.1007/s00198007-0349-5

25. Zethraeus N, Borgstrom F, Strom O, Kanis JA, Jonsson B. Costeffectiveness of the treatment and prevention of osteoporosis a review of the literature and a reference model. Osteoporos Int 2007; 18(1): 9-23. doi:10.1007/s00198-006-0257-0

26. Iglesias CP, Manca A, Torgerson DJ. The health-related quality of life and cost implications of falls in elderly women. Osteoporos Int 2009; 20(6): 869-78. doi:10.1007/s00198008-0753-5

27. Henry DA, Hill SR, Harris A. Drug prices and value for money: the Australian Pharmaceutical Benefits Scheme. JAMA 2005; 294(20): 2630-2. doi:10.1001/jama.294.20.2630

28. Swanenburg J, de Bruin ED, Stauffacher M, Mulder T, Daniel U. Effects of exercise and nutrition on postural balance and risk of falling in elderly people with decreased bone mineral density: randomized controlled trial pilot study. Clin Rehabil 2007; 21(6): 523-34. doi:10.1177/0269215507075206

29. Sherrington C, Lord S, Close J. Best practice recommendations for physical activity to prevent falls in older adults (unpublished report to the NSW Department of Health), 2008.

30. Clemson L, Cumming RG, Kendig H, Swann M, Heard R, Taylor K. The effectiveness of a community-based program for reducing the incidence of falls in the elderly: a randomized trial. J Am Geriatr Soc 2004; 52(9): 1487-94. doi:10.1111/ j.1532-5415.2004.52411.x

31. Becker C, Kron M, Lindemann U, Sturm E, Eichner B, WalterJung B et al. effectiveness of a multifaceted intervention on falls in nursing home residents. J Am Geriatr Soc 2003; 51(3): 306-13. doi:10.1046/j.1532-5415.2003.51103.x 